BMJ Open Sport \& Exercise Medicine

\title{
Video analysis of high-magnitude head impacts in men's collegiate lacrosse
}

\author{
Kari Kindschi, ${ }^{1}$ Michael Higgins, ${ }^{2}$ Andrea Hillman, ${ }^{3}$ Gregory Penczek, ${ }^{3}$ \\ Andrew Lincoln ${ }^{1}$
}

To cite: Kindschi K, Higgins M, Hillman A, et al. Video analysis of highmagnitude head impacts in men's collegiate lacrosse. BMJ Open Sport Exerc Med 2017;0:e000165. doi:10.1136/bmjsem-2016000165

Accepted 9 April 2017
CrossMark

\section{${ }^{1}$ Department of Sports Medicine, MedStar Union Memorial Hospital, Baltimore, Maryland, USA ${ }^{2}$ University of Virginia, Charlottesville, Virginia, USA ${ }^{3}$ Kinesiology Department, Towson University, Towson, Maryland, USA}

Correspondence to Dr Kari Kindschi; kari.e. kindschi@medstar.net

\section{ABSTRACT}

Background/aim Lacrosse is one of the fastest growing sports in the USA. Efforts to minimise head injuries focus on promoting safe play through player and coach education, rules enforcement and use of effective protective equipment. The study aims to determine event characteristics of high-magnitude head impacts in men's collegiate lacrosse competitions through video analysis.

Methods Seventeen Division I men's collegiate lacrosse players wore instrumented helmets that collected biomechanical measures of head impacts. During 15 competitions, the magnitude of linear acceleration, rotational velocity and helmet impact location were recorded. Impacts with linear accelerations above a $70 \mathrm{~g}$ threshold were correlated with video to confirm impact location and to determine event characteristics-source of impact and player activity at the time of impact.

Results A total of 122 high-magnitude impacts were reviewed on video. Player-to-player contact $(n=94$, $77.0 \%$ ) was the most common impact mechanism, followed by stick-to-player contact $(n=11,9.0 \%)$. Impacts occurred most often when the athlete was delivering a body check ( $n=39,32.0 \%$ ), fighting for loose ball possession ( $\mathrm{n}=35,28.7 \%)$ or attacking the goal $(n=35,28.7 \%)$. The most frequent impact locations were the front of the helmet $(n=46,37.8 \%)$ and the left side of the helmet $(n=26,21.3 \%)$.

Conclusions In men's collegiate lacrosse games, the majority of high-magnitude head impacts resulted from player-to-player contact when the sensored athlete did not have possession of the ball. Video analysis provides the game context for head impact mechanisms, which is critical to developing sportspecific injury prevention strategies.

\section{INTRODUCTION}

Men's lacrosse is the fastest growing team sport in America. ${ }^{1}$ The men's game is a contact sport and ranks in the middle of men's collegiate sports in terms of injury rate. ${ }^{2}$ Concussions represented $8.6 \%$ of injuries in games according to a study reviewing 16 years of National Collegiate Athletic Association (NCAA) men's collegiate lacrosse injury surveillance data. ${ }^{3}$ The concussion rate in competition (1.08/1000 athletic exposures) was higher than in

\section{What are the new findings}

This is the first study to pair helmet sensor data with video analysis of head impacts during collegiate lacrosse competitions.

- Most of the high-magnitude head impacts resulted from player-to-player contact when the sensored athlete did not have possession of the ball.

- A single high-magnitude impact (1/122, $0.8 \%)$ resulted in a head injury.

How might it impact on clinical practice in the near future

- Event characteristics and impact mechanisms may inform injury prevention strategies and aid in the evaluation of interventions, like rule changes, already in place.

practice $\left(0.12 / 1000\right.$ athletic exposures). ${ }^{3}$ Descriptive epidemiological studies have typically reported lacrosse injuries as an incidence per athletic exposures. It is difficult to assess individual exposure, event characteristics or injury mechanisms with this measure.

Little is known about the biomechanics of concussions, the relationship between head impacts and concussion risk, or the cumulative effects of subconcussive impacts. Epidemiological data describing head impact exposure or the mechanism of head injury in men's collegiate lacrosse players are limited. Accelerometer-based exposure studies of contact sports have largely focused on biomechanical data (linear and rotational acceleration of the head) in football $^{4-6}$ or ice hockey players. ${ }^{7}$ While the research identifies impact patterns and characteristics among individual athletes, it is unclear if the information translates to other helmeted sports.

Several sports have characterised injury mechanisms and head impacts through video review. Rugby studies detailed event 


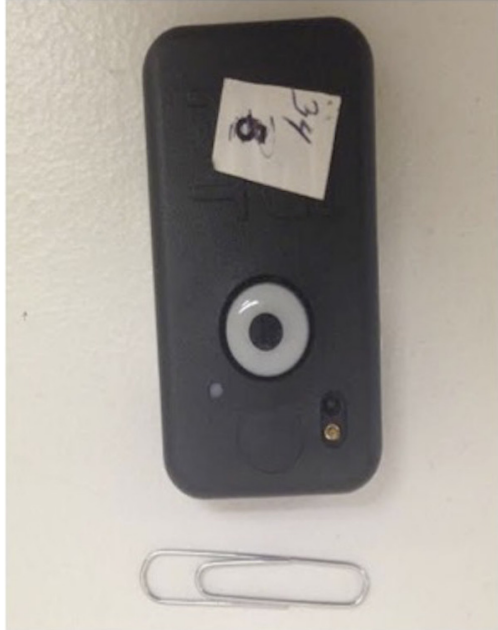

Figure 1 GForce Tracker sensor.

circumstances of concussive impacts and clarified both the site of impact and the striking object. ${ }^{8}{ }^{9}$ A baseball study described the helmet location of ball impacts as well as responder assessment after impact. ${ }^{10}$ Video analysis provided information about impact characteristics and injury mechanisms in ice hockey competitions. ${ }^{71}{ }^{12}$ Some of the earliest examples of video analysis in sport come from football where it was used to simulate collisions to determine impact biomechanics. ${ }^{13}$ Mixed martial arts research identified impact location and mechanism of knockouts through video review. ${ }^{14}$ In addition to demonstrating impact location, mechanism of injury and event descriptions in cricket, video analysis has also been used to evaluate helmet safety within the sport. ${ }^{15}$ Video review of head injury mechanisms in elite soccer matches highlighted game play scenarios with a high risk of head injuries. ${ }^{16}$ While this is the first study to use video analysis in men's collegiate lacrosse, it has characterised head injury events in both boys' and girls' high school lacrosse competitions. ${ }^{17} 18$

To address the gaps in knowledge surrounding individual player exposure and impact characteristics in men's collegiate lacrosse, this study sought to describe high-magnitude head impacts during competition through video analysis.

\section{METHODS}

\section{Participants}

Seventeen Division I NCAA men's lacrosse players (height $173.89 \pm 2.45 \mathrm{~cm}$; weight $72.65 \pm 3.32 \mathrm{~kg}$ ) volunteered to be part of this study. Participants gave written informed consent. The Institutional Review Board of Towson University approved the study. The study included two goalies, three defenders, six midfielders, two face off specialists and four attack players. The athletes ranged in age from 18 to 22 years. A concussion history was obtained from each athlete during a preseason physical examination.

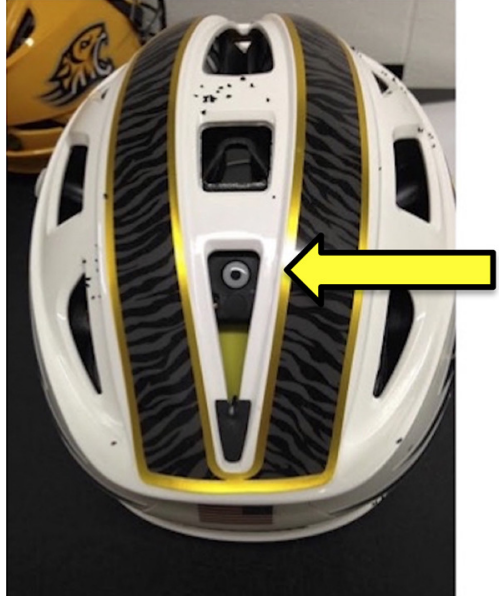

Figure 2 Sensor placement.

\section{Instrumentation}

All players were fitted with a Cascade $\mathrm{R}$ helmet at the beginning of the season. During the course of the year, a player used three helmets-one for practice, one for home games and one for away games. Each participant was assigned one GForce Tracker sensor (Hardware version: GFT3.s.19; Artaflex, Markham, Ontario, Canada). Depending on the type of session, the individual's sensor was transferred to the appropriate helmet. The device is an accelerometer-based linear $g$ force and rotational monitoring system. The sensor measures $29 \mathrm{~mm}$ x $55 \mathrm{~mm}$ x $14 \mathrm{~mm}$ (figure 1). The device was attached with Velcro to the inner lining of the helmet in between the padding. The sensors were not directly attached to the shell of the helmet (figure 2).

The sensors measured acceleration-time history, magnitude of both linear acceleration and rotational velocity, and location of the impact. Data were collected in $40 \mathrm{~ms}$ windows $-8 \mathrm{~ms}$ preimpact and 32 ms postimpact. The impact location was calculated using azimuth and elevation. Each impact was categorised into one of six locations on the helmet-back, front, right, left, bottom (base of the helmet chin piece) and top. GForce Tracker validity and reliability have been assessed with laboratory-based testing demonstrating a moderate to strong correlation with reference measures inside a Hybrid III headform. ${ }^{19} 20$

\section{Data collection protocol}

Data were collected from games over the 2014 lacrosse season. During the study period, there were 15 competitions. Before each game, a trained member of the sports medicine staff placed the sensors in the players' helmets. At the end of play, the sensors were removed and the data were downloaded to a cloudbased software system before charging in a docking station. Based on previous studies, ${ }^{13} 21$ we defined a high-magnitude impact as one with a linear acceleration above $70 \mathrm{~g}$. Game impacts above this threshold were highlighted for investigation. 
Table 1 Impact characteristics from video review of game impacts greater than $70 \mathrm{~g}, \mathrm{n}=122$

Frequency, $\mathrm{n}(\%)$

\begin{tabular}{|c|c|}
\hline \multicolumn{2}{|l|}{ Activity during impact } \\
\hline \multicolumn{2}{|l|}{ No ball possession } \\
\hline Loose ball situation & $35(28.7)$ \\
\hline Defending/Delivering a check & $39(32.0)$ \\
\hline Hit by shot or deflection & $4(3.3)$ \\
\hline Setting a pick & 4 (3.3) \\
\hline Face off & $2(1.6)$ \\
\hline \multicolumn{2}{|l|}{ With ball possession } \\
\hline Attacking & $35(28.7)$ \\
\hline Preparing to pass/clear the ball & $3(2.5)$ \\
\hline \multicolumn{2}{|l|}{ Helmet impact location } \\
\hline Front & $46(37.8)$ \\
\hline Back & $9(7.4)$ \\
\hline Bottom & $13(10.7)$ \\
\hline Top & $13(10.7)$ \\
\hline Right & $15(12.3)$ \\
\hline Left & $26(21.3)$ \\
\hline \multicolumn{2}{|l|}{ Impact source } \\
\hline Stick & $11(9.0)$ \\
\hline Ball & $4(3.3)$ \\
\hline Opponent's body & $94(77.0)$ \\
\hline Opponent's helmet & $9(7.4)$ \\
\hline Ground & $4(3.3)$ \\
\hline
\end{tabular}

Video review was performed to determine circumstances of the impact, to identify what hit the athlete (ball, stick, another player, ground) and to confirm impact location. Video from each competition was recorded from the press box at the midfield line in a wide-angle format. Each impact was time stamped within the data set by the GForce Tracker. We confirmed sensor synchronisation with real time through correlation with the recorded start time for each quarter and the time of goals during the competition. Impacts above the $70 \mathrm{~g}$ threshold were identified within the game footage by the time stamp. Injury surveillance was performed throughout the study period with particular attention to head injuries.

\section{Video analysis protocol}

The protocol for video analysis was adapted from an incident analysis protocol for boy's lacrosse developed by Lincoln $\mathrm{et} \mathrm{al.}{ }^{17}$ High-magnitude impacts in competitions were reviewed on video through the TeamXstream video service. Two investigators independently analysed the videos to identify the impacts on video and to confirm the impact location with sensor data. Additionally, high-magnitude impacts were coded to describe player activity at the time of impact and impact source. Each impact was reviewed at normal speed and at a frame-by-frame speed. Descriptive statistics and $\chi^{2}$ tests were used to characterise the data.

\section{RESULTS}

\section{Impact characteristics}

During the study, sensors measured 162 game impacts above $70 \mathrm{~g}$ and 122 were confirmed by video. Twenty-eight impacts occurred during a warmup or post-game period when video was not recorded. We were unable to identify 12 of the impacts on video because the player was not in camera view at the time of impact.

Characteristics of game impacts greater than $70 \mathrm{~g}$ are summarised in table 1. Video analysis revealed that the majority of impacts were associated with loose ball situations, athletes attacking the goal or delivering a body check on defence. The most common impact location was the front of the helmet. Seventy-seven per cent of impacts above the threshold were the result of contact with the body of an opponent.

A $\chi^{2}$ test for one proportion was used to determine significance. The $\chi^{2}$ value for comparison of impacts without ball possession and with ball possession is 34.354 , p value $<0.0001$. For helmet impact location,

Table 2 High-magnitude impacts in competition as a function of position

\begin{tabular}{lcccc}
\hline Position & $\begin{array}{l}\text { Players } \\
\text { with sensors (n) }\end{array}$ & Impacts by position & Total impacts (\%) & Impacts per player \\
\hline Goalie & 2 & 5 & 4.1 & 2.5 \\
Defenders & 3 & 37 & 30.3 & 12.3 \\
Midfielders & 6 & 40 & 32.8 & 6.7 \\
Face off & 2 & 22 & 18.0 & 11.0 \\
Attack & 4 & 18 & 14.8 & 4.5 \\
\hline
\end{tabular}



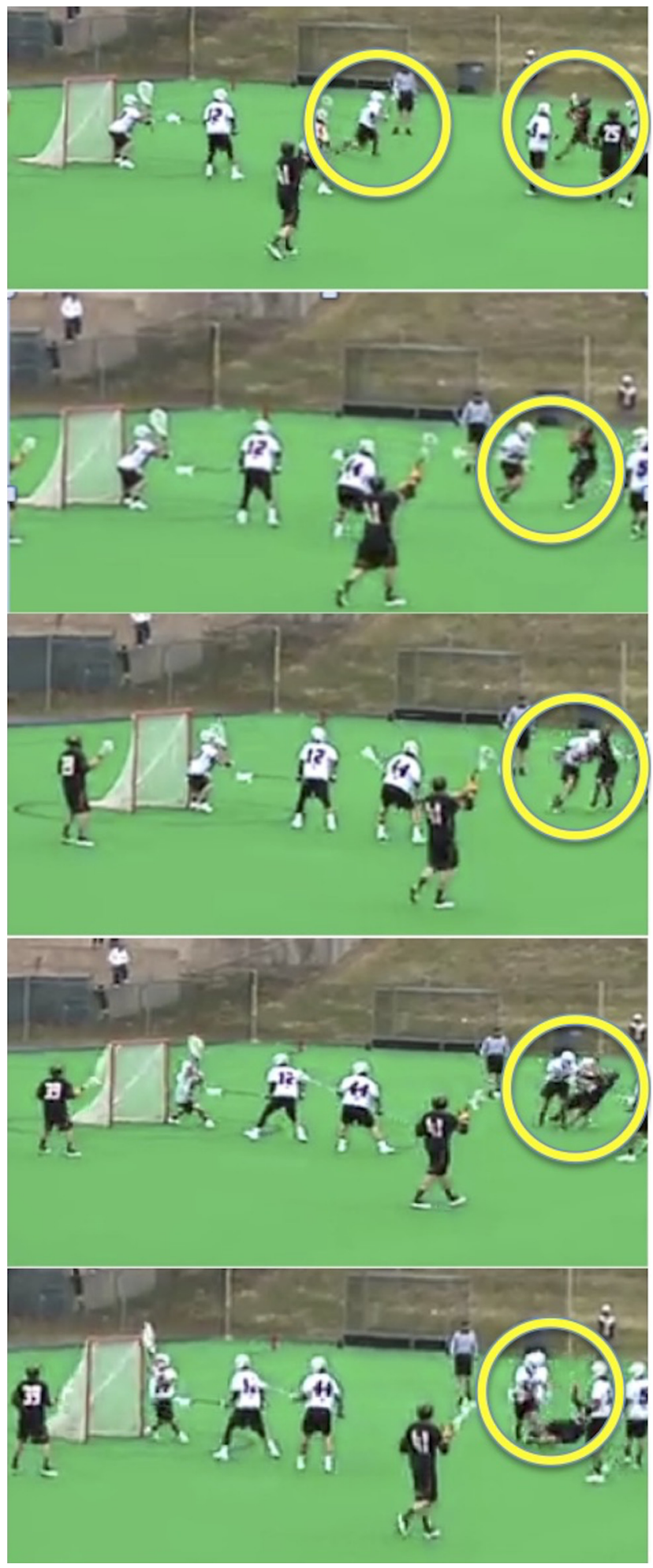

Figure 3 A player at the top right corner of the frame is checked while attacking the goal.

the $\chi^{2}$ value comparing front and left is 7.945 , $\mathrm{p}=0.0048$, while the $\chi^{2}$ value comparing left and right is 3.520 , $\mathrm{p}$ value $=0.0606$.

Player activity at the time of impact varied by position. Midfielders had almost twice as many highmagnitude impacts while attacking the goal compared with attack players. Impacts above $70 \mathrm{~g}$ during loose ball situations and while defending/ delivering a check were more common among defenders.

Table 2 characterises high-magnitude impacts in competition as a function of position. Per player, defenders had the most impacts above the $70 \mathrm{~g}$ threshold (12.3), followed by face off specialists, ${ }^{11}$ 
midfielders (6.7), attack athletes (4.5) and goalies (2.5).

\section{Injury surveillance}

Only one head injury (facial laceration) occurred during competition. The injury was associated with an impact above $70 \mathrm{~g}$. Figure 3 represents the frame-byframe game video of the player-to-player contact which led to injury.

\section{DISCUSSION}

\section{Impact characteristics}

We used video analysis to review the circumstances of head impacts above a $70 \mathrm{~g}$ linear acceleration threshold. The majority of impacts were located on the front of the helmet. The number of impacts to the left side of the helmet was nearly double the number of impacts to the right side. While most of our collegiate lacrosse athletes are comfortable with their stick in either hand, players have a natural tendency towards a preferred stick side. In this group of athletes, that preferred side correlated closely with handedness. Fifteen of the seventeen athletes in the study are right handed. With the stick on the right side of the body, the left side is more exposed, which may explain the greater number of left-sided high-magnitude impacts. The statistical difference between impacts to the left and right approached significance but was limited by the small sample size and therefore hints at, rather than confirms, a correlation between head impacts and handedness. This study highlights the need for further focused evaluation of head impact exposure in lacrosse as a function of handedness.

Not only could we confirm impact location with sensor data but we were also able to collect data on the mechanism of impact. In this study, $68.8 \%$ of impacts occurred when the sensored athlete did not have possession of the ball. Impacts while the athlete was defending or delivering a check represented the most frequent activity at the time of the event. Most of the high-magnitude impacts captured on video (77\%) were the result of contact with an opponent's body.

\section{Position-specific characteristics}

Not surprisingly, activity at the time of impact was a function of player position. Defenders were more likely to have high-magnitude impacts in loose ball situations or while defending/delivering a check. Midfielders had the most impacts in attacking situations. All of the impacts resulting from a shot or a deflection were sustained by the goalies.

\section{Video analysis}

Video analysis has been used in rugby, baseball, football, mixed martial arts, soccer, hockey and cricket to describe head injury mechanisms and event characteristics. $^{8-16}$ This is the first study to use video in collegiate lacrosse, but it has been used to evaluate head injury events in high school boy's and girl's lacrosse. ${ }^{17} 18$ A study by Lincoln et al found that playerto-player contact was the primary mechanism of concussions in boy's high school lacrosse. ${ }^{17}$ While we did not have any concussions in competitions during the study period, the one head injury (laceration) in a game was the result of player-to-player contact. The source of the majority of high-magnitude impacts was an opponent's body, which is consistent with previous lacrosse studies at the high school level.

\section{Limitations and future directions}

The study captures data from one team, over one competitive season. Further study, incorporating several teams over several seasons, is needed to inform injury prevention strategies and drive policy changes in the sport of lacrosse.

Video was only available for competitions, and therefore we were unable to analyse high-magnitude impacts in practice settings. Expanding the study to include practice and warm-up periods will be important for further investigations. The high-magnitude impact classification for this study was based on a linear acceleration threshold of $70 \mathrm{~g}$. Future studies will benefit from broadening the biomechanical threshold to include a rotational velocity component.

There were no concussions in games during the spring season. With only one head injury captured on video, we were unable to link impact characteristics to injury mechanisms which makes it difficult to use this data to inform injury prevention strategies. While the lack of head injuries during the study period limits an evaluation of the mechanism of injury, our data may be better suited to analyse the 'mechanism of no injury' as introduced by Dr Willem Meeuwisse. ${ }^{22}$ He proposed studying the question: "why did an injury NOT occur?"22 in order to identify variables contributing to injury-free athletic movement and play. ${ }^{22}$ By comparing the mechanism of injury to the mechanism of no injury, we may be able to identify the critical event characteristic causing injury. ${ }^{22}$

Presently, injury control efforts are focused on promoting safe play through player and coach education, rules enforcement and the use of effective protective equipment. Analysing video to determine if the player was defenceless at the time of impact will be important to identify at-risk situations in future studies. The NCAA instituted rule changes to penalise targeting of the head and neck in men's lacrosse. ${ }^{23}$ It will take several seasons to know if the penalties are a deterrent and actually decrease the number of dangerous impacts. Future investigations are warranted to determine how well the referees are enforcing the rules. 


\section{CONCLUSION}

This is the first study to pair helmet sensor data with video analysis of head impacts during collegiate lacrosse competitions. The study revealed that most high-magnitude impacts resulted from player-toplayer contact when the sensored athlete did not have possession of the ball. Only one impact above the $70 \mathrm{~g}$ threshold was associated with head injury. Frequency of high-magnitude impacts and player activity at the time of impact were a function of position.

Video analysis serves as an important adjunct to numerical sensor data. It enables investigators to determine event characteristics and impact mechanisms that are critical to developing sport-specific head injury prevention strategies and evaluating the effectiveness of interventions, like rule changes, already in place.

Acknowledgements The authors would like to thank the dedicated certified athletic trainers and student athletic trainers at Towson University who provided assistance in data collection.

Contributors Each author was involved in the planning, conducting and reporting of this work. $\mathrm{KK}$ is the guarantor and is responsible for the overall content.

Competing interests None declared.

Ethics approval Towson University Institutional Review Board.

Provenance and peer review Not commissioned; externally peer reviewed.

Open Access This is an Open Access article distributed in accordance with the Creative Commons Attribution Non Commercial (CC BY-NC 4.0) license, which permits others to distribute, remix, adapt, build upon this work noncommercially, and license their derivative works on different terms, provided the original work is properly cited and the use is non-commercial. See: http:// creativecommons.org/licenses/by-nc/4.0/

(C) Article author(s) (or their employer(s) unless otherwise stated in the text of the article) 2017. All rights reserved. No commercial use is permitted unless otherwise expressly granted.

\section{REFERENCES}

1. US Lacrosse. Participation study. 2012. Available at http://www. uslacrosse.org/about-the-sport/participation-survey.aspx (accessed 25 Mar 2014).

2. Kerr ZY, Marshall SW, Dompier TP, et al. College sports-related injuries-United States, 2009-10 through 2013-14 academic years. MMWR Morb Mortal Wkly Rep 2015;64:1330-6.
3. Dick R, Romani WA, Agel J, et al. Descriptive epidemiology of collegiate men's lacrosse injuries: National Collegiate Athletic Association Injury Surveillance System, 1988-1989 through 2003-2004. J Athl Train 2007:42:255-61.

4. Crisco JJ, Fiore R, Beckwith JG, et al. Frequency and location of head impact exposures in individual collegiate football players. $J$ Athl Train 2010;45:549-59.

5. Crisco JJ, Wilcox BJ, Beckwith JG, et al. Head impact exposure in collegiate football players. J Biomech 2011;44:2673-8.

6. Crisco JJ, Wilcox BJ, Machan JT, et al. Magnitude of head impact exposures in individual collegiate football players. $J$ Appl Biomech 2012;28:174-83.

7. Wilcox BJ, Machan JT, Beckwith JG, et al. Head-impact mechanisms in men's and women's collegiate ice hockey. J Athl Train 2014;49:514-20.

8. Gardner AJ, Iverson GL, Quinn TN, et al. A preliminary video analysis of concussion in the National Rugby League. Brain Inj 2015;17:1182-5.

9. Mclntosh AS, McCrory P, Comerford J. The dynamics of concussive head impacts in rugby and Australian rules football. Med Sci Sports Exerc 2000;32:1980-4.

10. Athiviraham A, Bartsch A, Mageswaran P, et al. Analysis of baseballto-helmet impacts in major league baseball. Am J Sports Med 2012;40:2808-14.

11. Hutchison MG, Comper $\mathrm{P}$, Meeuwisse $\mathrm{WH}$, et al. A systematic video analysis of National Hockey League (NHL) concussions, part I: who, when, where and what? Br J Sports Med 2015:49:547-51.

12. Hutchison MG, Comper $\mathrm{P}$, Meeuwisse $\mathrm{WH}$, et al. A systematic video analysis of National Hockey League (NHL) concussions, part II: how concussions occur in the NHL. Br J Sports Med 2015;49:552-5.

13. Pellman EJ, Viano DC, Tucker AM, et al. Concussion in professional football: reconstruction of game impacts and injuries. Neurosurgery 2003;53:799-814.

14. Hutchison MG, Lawrence DW, Cusimano MD, et al. Head trauma in mixed martial arts. Am J Sports Med 2014;42:1352-8.

15. Ranson C, Peirce N, Young M. Batting head injury in professional cricket: a systematic video analysis of helmet safety characteristics. Br J Sports Med 2013;47:644-8.

16. Andersen TE, Arnason A, Engebretsen L, et al. Mechanisms of head injuries in elite football. Br J Sports Med 2004;38:690-6.

17. Lincoln AE, Caswell SV, Almquist JL, et al. Video incident analysis of concussions in boys' high school lacrosse. Am J Sports Med 2013:41:756-61.

18. Caswell SV, Lincoln AE, Almquist JL, et al. Video incident analysis of head injuries in high school girls' lacrosse. Am J Sports Med 2012;40:756-62.

19. Allison MA, Kang YS, Maltese MR, et al. Measurement of Hybrid III head impact kinematics using an accelerometer and gyroscope system in ice hockey helmets. Ann Biomed Eng 2015;43:1896-906.

20. Campbell KR, Warnica MJ, Levine IC, et al. Laboratory evaluation of the gForce Tracker ${ }^{T M}$, a head impact kinematic measuring device for use in football helmets. Ann Biomed Eng 2016;44:1246-56.

21. Zhang L, Yang KH, King Al. A proposed injury threshold for mild traumatic brain injury. J Biomech Eng 2004;126:226-36.

22. Meeuwisse WH. What is the mechanism of no injury (MONI)? Clin $J$ Sport Med 2009;19:1-2.

23. National Collegiate Athletic Association. NCAA Men's Lacrosse Rules. 2013-14. Available at http://www.ncaapublications.com/p4279-2013-and-2014-mens-lacrosse-rules-and-interpretationsupdated-feb-2014.aspx (accessed 7 Apr 2014). 